\title{
A negação não psicótica da gravidez: vicissitudes de um não saber
}

\section{The non-psychotic denial of pregnancy: vicissitudes of a not knowing}

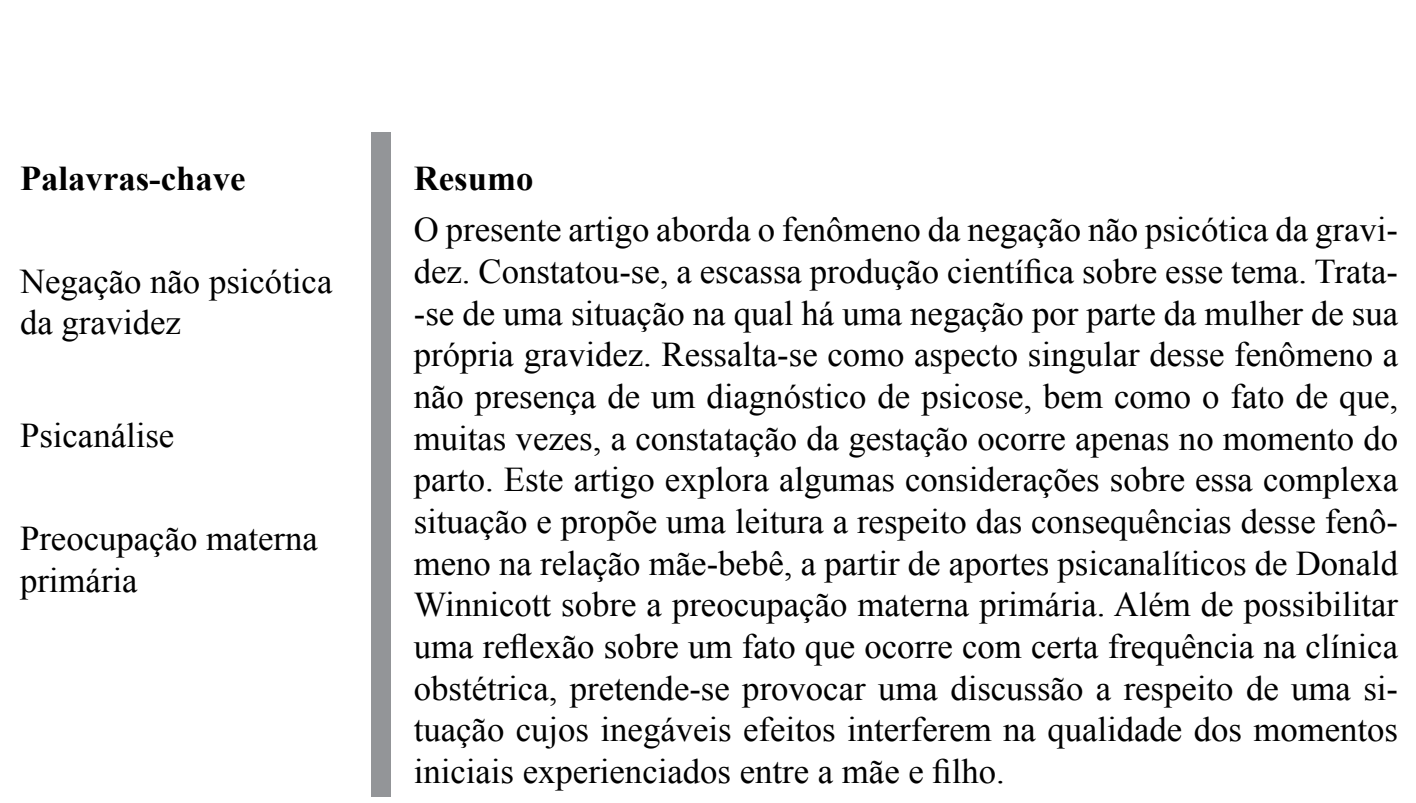

\section{Abstract}

This article addresses the phenomenon of non-psychotic denial of pregnancy. It was realized that there is a few scientific production about this topic. It is about a situation which there's a denial from woman of her own pregnancy. It is highlighted as a singular aspect about the absence of a psychosis diagnostic, as well as, the fact that in many cases, that some women realize that they are pregnant just when they are in labor. This paper explores some considerations about this complex situation and proposes a lecture about the consequences of this phenomenon in the relationship between mother and child from psychoanalytic support by Donald Winnicott about the Primary Maternal Preoccupation. Besides enabling some thoughts about the fact which occurs with some frequency in the obstetrical clinic, it is intended to discuss about this situation which the undeniable effects interfere in the primary quality moments experienced between mother and child.

\section{Keywords}

Non-psychotic denial of pregnancy

psychoanalysis

primary maternal preoccupation

\footnotetext{
1. Psicólogo. Mestre em Psicologia Clínica pelo Programa de Pós-graduação em Psicologia na Pontifícia Universidade Católica do Rio Grande do Sul (PUCRS). Doutorando em Psicologia do Desenvolvimento pela Universidade Federal do Rio Grande do Sul. Membro da Associação Francesa pelo Reconhecimento da Negação da Gravidez (Toulouse, França). Psicanalista em formação pelo Centro de Estudos Psicanalíticos de Porto Alegre.
} 


\section{Introdução}

A situação complexa de não reconhecimento da própria gravidez por parte de mulheres sem diagnóstico de psicose tem sua compreensão pouco explorada em artigos científicos. Trata-se de uma situação denominada em inglês como non psychotic denial of pregnancy. Nessa situação, ocorre um fenômeno de negação da própria gravidez, por parte de mulheres não psicóticas. Esse fenômeno pode ser definido como a falta de consciência subjetiva por parte da mulher em relação a estar grávida (WESSEL; ENDRIKAT; BUSCHER, 2002). Corroborando com essa asseveração, o termo "negação da gravidez" refere-se também à ignorância, por parte da mulher, de seu estado gravídico (FERRAGU, 2002). Na negação não psicótica da gravidez, a realidade está preservada, ou seja, a realidade não é substituída por outra realidade alternativa, logo as grávidas não estão vivenciando um episódio psicótico (SPIELVOGEL; HOHENER, 1995). Esse fenômeno, então, não é nem uma dissimulação da gravidez e tampouco resultante de uma condição psíquica de ruptura com a realidade. Não se trata, portanto, de uma situação na qual a mulher sabe que está grávida, porém não revela esse fato aos demais, por medo do que a gravidez possa causar em seu entorno.

Constatou-se, porém, mediante busca e leitura de artigos científicos nacionais e internacionais, que são escassos os estudos realizados sobre essa temática. No Brasil não foi encontrada nenhuma produção sobre o tema. Logo, parece ser inquestionável a importância de abrir um espaço de reflexão e de estudo sobre tão relevante situação (GONÇALVES; MACEDO, 2012).

Dessa forma, trata-se de um tema bastante importante no que diz respeito à experiência de maternidade, a qual tem e terá consideráveis efeitos psíquicos, tanto para a mulher frente ao nascimento de seu filho, quanto para a saúde emocional do bebê. Todos esses fatores motivaram o presente estudo sobre o fenômeno da non psychotic denial of pregnancy.

\section{Non psychotic denial of pregnancy - considerações iniciais}

Para melhor compreensão do fenômeno da negação não psicótica da gravidez, é necessário situá-lo como não raro e tampouco exótico, contrariando, assim, o senso comum. O que chama atenção em termos de cada vez mais rara aparição diz respeito à gravidez psicológica, ou seja, pouco surgem na clínica situações desta natureza na qual uma mulher pensa estar grávida sem realmente estar (WESSEL; ENDRIKAT; BUSCHER, 2002). Além disso, a situação da ocorrência da negação não psicótica da gravidez é mais frequente do que a eritroblastose fetal e a ruptura uterina; sendo, ainda, três vezes mais comum do que o nascimento de trigêmeos (WESSEL; ENDRIKAT; BUSCHER, 2002).

Um dos fatores que contribuem para que a mulher negue a situação de estar grávida é a ocorrência de uma suposta menstruação. Em estudos realizados sobre a negação não psicótica da gravidez, várias mulheres reportaram ter apresentado uma menstruação contínua, sendo que, em verdade, seria um sangramento que se confundiria com a menstruação (WESSEL; ENDRIKAT, 2005). A causa desse sangramento que leva a mulher a crer que esteja acontecendo uma menstruação regular ainda não é bem conhecida. Pode se tratar de um endométrio cervical instável ou de algo relacionado à cérvix como fonte do sangramento, ou ainda algo relativo à pseudo-menstruação advinda da decídua (WESSEL; ENDRIKAT, 2005). Apesar de divergirem sobre a sua origem, os autores concordam ao afirmar que o sangramento que ocorre na negação não psicótica da gravidez não é o sangramento de uma menstruação normal.

Quando nos deparamos com o fenômeno da non psychotic denial of pregnancy, é necessário refletir sobre os fatores de risco tanto para a mãe, quanto para o bebê. Os fatores de risco que concernem à negação não psicótica da gravidez denunciam certa discordância entre autores estudiosos dessa temática. Por um lado, alguns deles colocam como fatores de risco: o isolamento social, baixo poder aquisitivo, inteligência baixa, imaturidade, baixa idade, concepção premarital, a condição feminina de ser solteira e, até mesmo, a ingenuidade em relação 
às funções do corpo (NEIFERT; BOURGOIS, 2000). Por outro lado, certos achados contradizem esses fatores de risco, pois, em pesquisa com 65 casos de mulheres que apresentaram non psychotic denial of pregnancy, apenas nove tinham menos de 20 anos, mais de $80 \%$ possuíam um relacionamento estável e tinham ensino completo, sendo que apenas um terço das participantes do estudo não tinha estado grávida anteriormente (WESSEL, 2007). Além dessas refutações, os autores afirmam que alguns fatores de risco são insuficientes para identificação da maioria das mulheres que negaram a situação da gravidez, enfatizando, no entanto, a heterogeneidade como uma característica do fenômeno.

A Portaria GM/MS n ${ }^{\circ}$ 569/GM, de $1^{\circ}$ de junho de 2000, do Ministério da Saúde do Brasil, estabeleceu o Programa de Humanização no Pré-natal e Nascimento (PHPN), tendo como objetivo estimular que municípios e estados possibilitem um melhor acompanhamento às mulheres grávidas no cadastramento, nos exames da etapa pré-natal, ampliação da cobertura, melhoria no acesso aos serviços de saúde para as gestantes, buscando, assim, diminuir as taxas de morbimortalidade materna e perinatal (MINISTÉRIO DA SAUDE, 2000). No PHPN, vários exames laboratoriais e testes são preconizados, como, por exemplo, o VDRL (Venereal Disease Research Laboratory), que é um teste para identificação de pacientes com sífilis, além de testagem anti-HIV, exame de hemoglobina, hematócrito, etc. Trata-se de uma iniciativa do Ministério da Saúde que reconhece a importância e visa atender necessidades decorrentes da condição de gravidez.

Logo, considerando-se a relevância de um acompanhamento pré-natal, um dos inquestionáveis riscos na situação de negação não psicótica da gravidez é o fato dessas mulheres não terem realizado nenhum exame de acompanhamento durante boa parte ou toda a sua gravidez. O processo inteiro de acompanhamento e os exames citados deixaram de ser realizados devido a esta situação de não reconhecimento da gravidez por parte dessas mulheres, que colocam a si mesmas e ao feto em situação de risco. O fato de a gestante estar fazendo o pré-natal possibilita vários benefícios, tanto para ela mesma quanto para seu filho, sendo que este acaba por apresentar um melhor crescimento intrauterino, assim, como menor mortalidade perinatal e infantil (HALPERN ET AL., 1998). O acompanhamento pré-natal é referido como um dos fatores que contribuem para que não haja uma taxa maior de mortalidade perinatal (TREVISAN ET AL., 2002). Alguns riscos são apontados para o recém-nascido na ocorrência de negação da gravidez, como a prematuridade e baixo peso na idade gestacional (WESSEL; ENDRIKAT; BUSCHER, 2002). Nessa direção, existe a necessidade de se fazer uma avaliação pediátrica, além de se ter um apoio do serviço social e uma avaliação da capacidade de cuidar de um filho para todas as mulheres que não fizeram o pré-natal (FRIEDMAN, HENEGHAN; ROSENTHAL, 2007).

Para que esta situação de negação da gravidez tenha ocorrido, pode-se supor que também alguns procedimentos por parte dos médicos tenham deixado a desejar. É o caso de que os médicos considerem, em seu exercício clínico, a possibilidade de uma negação não psicótica da gravidez frente à necessidade de um diagnóstico diferencial, ou seja, para mulheres que estejam apresentando náuseas, dores abdominais, aumento de peso, e tendo ou não amenorreia, deveria ser considerada a possibilidade de negação da gravidez (FRIEDMAN, HENEGHAN; ROSENTHAL, 2007).

Outra consequência possível frente ao fenômeno da negação não psicótica da gravidez é a ocorrência do neonaticídio. Para melhor compreensão da dinâmica própria dessa situação, é necessário diferenciar o neonaticídio do infanticídio e do filicídio. Os três fenômenos são assassinatos praticados por um ou por ambos os pais contra seus filhos. O neonaticídio é a morte de um recém-nascido nas primeiras 24 horas de vida, já o infanticídio se caracteriza pela morte de uma criança até um ano de idade e o filicídio é a morte de indivíduos com idade superior a um ano (RESNICK, 1969). Não há nenhum crime de compreensão mais complexa do que o assassinato de um bebê pelo seu progenitor (RESNICK, 1970). São descritos dois tipos diferentes de neonaticídio: ativo e passivo. $\mathrm{O}$ primeiro se refere a uma violência direta relacionada a um pânico extremo, e o segundo a uma negligência praticada contra o bebê logo após o parto (BONNET, 1993). 
As motivações para que um progenitor mate seu filho são muito diferentes no neonaticídio, quando se compara ao filicídio. No neonaticídio o motivo aparente mais comum é o de filho não desejado, com $83 \%$, enquanto que no filicídio essa porcentagem cai para $11 \%$; em relação a uma motivação altruísta, ou seja, nos casos de transtorno de depressão maior, depressões psicóticas e psicose, no filicídio essa motivação é a predominante, com 53\%, enquanto que no neonaticídio esse número cai para $3 \%$ (BONNET, 1993).

É importante ressaltar que as mães que cometem neonaticídio não apresentam transtornos mentais significativos (BONNET, 1993). O neonaticídio não está relacionado com a tristeza materna (baby blues), pois esta ocorre aproximadamente três dias depois do parto (DOBSON; SALES, 2000). O neonaticídio não é um ato premeditado e, sim, uma resposta a uma situação de forte intensidade, tal como a culpa, o medo e o choque (PITT; SALE, 1995).

Por isso, é importante que o fenômeno da negação não psicótica da gravidez seja encarado como uma situação que efetivamente pode acontecer e, ainda, tendo uma frequência até mesmo maior do que a inicialmente pensada por leigos e profissionais de modo geral. A intervenção adequada por parte de profissionais que lidam diretamente com mulheres e com situações de ocorrência de uma gravidez pode evitar as graves consequências decorrentes desse fato, como, por exemplo, as importantes dificuldades emocionais nestes tempos iniciais da relação mãe-bebê.

\subsection{A negação não psicótica da gravidez: reflexões a partir de Donald Winnicott}

Algumas considerações quanto aos aspectos emocionais são importantes na aproximação e estudo do fenômeno da negação da gravidez não psicótica. Nesse sentido, encontram-se, nos aportes psicanalíticos de Donald Winnicott, elementos que contribuem para a exploração das complexidades psíquicas envolvidas nessa situação. O conceito de Preocupação Materna Primária (WINNICOTT, 1950) refere-se a um estado de grande sensibilidade em que se encontra a mãe durante e, principalmente, no final da gravidez. Em inglês, o termo para Preocu- pação Materna Primária é Primary Maternal Preoccupation. É interessante analisar a definição sobre o verbete preoccupation. Segundo o dicionário AURÉLIO (2007), preoccupation refere-se a um estado de pensamento contínuo sobre alguma coisa; algo que o sujeito pensa frequentemente, ou por um longo tempo, ou ainda um estado de espírito criado pelo pensamento que resulta em cuidado sobre alguma coisa e em ignorância quanto a outras. Assim, a pessoa que está preocupada não presta atenção nas demais coisas, pelo contínuo pensamento e inquietação sobre algo.

$\mathrm{Na}$ preocupação de uma mãe preocupada não se trata de algo corriqueiro, ou seja, não é uma preocupação qualquer, e sim uma preocupação que passa pelos cuidados que despende a seu filho recém-nascido. É uma preocupação que envolve o acalmar, aquecer o corpo do bebê, amamentá-lo e consolá-lo em suas dores (GRANATO, 2000). A Preocupação Materna Primária é definida como uma condição que a mãe deve alcançar, no início mesmo da vida do bebê, que corresponde a uma sensibilidade exacerbada sobre tudo o que diga respeito a seu filho (WINNICOTT, 1950). Essa condição se prolonga por algumas semanas após o parto e, não fosse o fato da própria gravidez, esse estado seria considerado uma doença, devido às suas características de retraimento, dissociação e fuga, podendo ser comparado também a um estado mais profundo, como um episódio esquizoide(WINNICOTT, 1950). Além disso, segundo DIAS (2003), este estado em que se encontra a mãe é de extrema importância para o bem-estar do lactente. Se existe uma mãe preocupada, há um bebê que está numa continuidade de ser.

É relevante aqui diferenciar o que é da ordem da espontaneidade e o que é da ordem da reatividade. Nesse sentido, o bebê é pensado como uma bolha. Se a pressão externa e a pressão interna estão equalizadas, este bebê, esta bolha, pode seguir na sua existência, este bebê pode continuar sendo. Caso a pressão externa e a interna estejam descompassadas, haverá uma reação à intrusão (DIAS, 2003). Essa bolha se modifica como reação a uma mudança no ambiente, não passando por um impulso próprio. A autora refere que, nessa situação, “o bebê terá uma interrupção do ser, produzida pela reação à intrusão" (DIAS, 2003, p.159). 
Este fenômeno da Preocupação Materna Primária proporciona uma situação na qual alguém está suficientemente identificado com o bebê, identificando, desse modo, suas demandas e dando condições de continuidade de ser ao bebê. Em relação a esta condição de identificação, as mães vão, gradativamente, ainda com seu bebê no útero, tendo uma percepção aguçada perante as necessidades do filho que está sendo gestado (WINNICOTT, 1979). Essa identificação permanece por algum tempo após o parto e vai perdendo sua relevância aos poucos. Sem essa identificação, a mãe não estaria atendendo aquilo que é necessário no começo da vida seu filho, ou seja, uma adaptação viva às necessidades do recém-nascido (WINNICOTT, 1979). Porém, um aspecto que merece ser destacado é a menção feita por Winnicott ao fato de que a mãe deve ter saúde suficiente para desenvolver este estado de doençalenamoramento e, também, para recuperar-se dele à medida que o bebê a libere (MOREIRA, 2007).

Por mais que a preocupação materna primária esteja atrelada à condição de maternidade, há mulheres que, de alguma forma, evitam entrar em contato com esse tipo de estado, ou seja, evitam se submeter à regressão que existe nessa condição; não conseguindo assim se direcionar e se focar no bebê que esperam, não obtendo, dessa forma, uma identificação com o bebê (DIAS, 2003). Nas situações em que as mães que não estabelecem uma comunicação profunda com os seus filhos, por estarem ocupadas com outras preocupações, há o sério risco de que elas não consigam cuidar do lactente de forma adequada, fazendo-o somente, então, por via mental, de maneira engessada, intelectualizada e deliberada. Neste modelo de relação, provavelmente, a mãe seguirá apenas roteiros de como se deve cuidar de um bebê, tendo, assim, um contato impessoal, não estabelecendo um cuidado suficientemente bom (DIAS, 2003).

Associando, portanto, o conceito de Preocupação Materna Primária com a situação de negação da gravidez em mulheres não psicóticas, pode-se apresentar algumas importantes considerações. Tomando como ponto de partida a constatação de que essas mulheres não sabiam que estavam grávidas, cabe a reflexão a respeito do quanto a sua relação com o bebê pode ficar prejudicada. Justamente por essas mães não terem estado nessa condição de sensibilidade, tão necessária para uma adaptação melhor do lactente ao mundo, cabe a preocupação com os efeitos prejudiciais daí decorrentes tanto para a mãe, quanto para seu filho. Esta provisão ambiental tão essencial para o desenvolvimento maturativo das primeiras semanas e meses da vida do bebê, quando falha, pode constituir-se em um fator traumático que terá importante repercussão no processo de integração que favorece ou não a formação de self (WINNICOTT, 1979). Quando não há essa falha, o bebê vive de maneira subjetiva, por um certo período de tempo, num mundo onde a realidade externa não está incidida; e este bebê vive "um senso de predizibilidade, e, desta maneira, as funções dos estágios iniciais muito delicados do crescimento da personalidade podem ser assentadas. Profilaxia, no contexto da saúde mental, é a provisão de uma facilitação suficientemente boa neste estágio inicial" (WINNICOTT, 1979, p.220). Este senso de predizibilidade é "uma espécie de conhecimento baseado na familiaridade, que torna possível ao bebê ser capaz de reter lembranças, esperar pelos cuidados e cogitar a respeito dos sentimentos da mãe. Desse modo, é a previsibilidade dos cuidados maternos que viabiliza o encadeamento paulatino de um sentido de passado, presente e futuro e a conformação do eu enquanto uma unidade" (MELLO, 2008, p.44).

Pode-se pensar, portanto, na relevância de um momento anterior a esse, ou seja, a vida intrauterina. Como se dá o acompanhamento/ experiências do período pré-natal e quais as possíveis consequências da não consciência da mãe perante a sua própria gestação?

Em algum momento da gestação o bebê tem um primeiro despertar, ou seja, uma consciência ainda muito primitiva, assim como um incipiente estado de ser e da continuidade de sua existência no tempo. Os movimentos que o feto faz vão sendo retidos por memórias corporais e, aos poucos, a junção dessas memórias vai vingar num novo ser humano. Quando o movimento parte de dentro para fora, ou seja, a ação advém do bebê e a mãe consegue respeitar essa exploração que o bebê está fazendo do ambiente, isso ajudará a estabelecer um padrão de relacionamento. Por outro lado, se o movimento parte do externo para o interno, isto é, se o ambiente se antecipa ao movimento do bebê, ocor- 
rerá uma intrusão nesta tentativa de criação de um padrão de relacionamento(DIAS, 2003).

$\mathrm{O}$ bebê pode ser atingido, ainda na vida intrauterina, por uma rigidez ou inadaptabilidade advindas da ansiedade ou de um estado depressivo materno (WINNICOTT, 1988). Cabe também ressaltar que "o feto pode sentir mudanças repentinas no ritmo cardíaco da mãe, bem como suas movimentações bruscas ou os efeitos de uma alimentação tóxica ou desregrada" (WINNICOTT, 1979, p.159). Logo, deve-se considerar o prejuízo que pode sofrer o feto quando a mãe não está consciente de estar gestando um ser que necessita, desde essa época, de um cuidado especial.

Há três maneiras mediante as quais se dá a comunicação entre a mãe e seu feto: por meio empático, fisiológico, além do comportamental. Por via fisiológica, segundo a autora, a mãe vincula suas emoções ao bebê e, além disso, qualquer outra substância de que a mãe faça uso irá passar diretamente para o feto, como, por exemplo, o álcool, o fumo, drogas, etc. Já, por via empática, a mãe se vincula ao desenvolvimento afetivo do feto, estando afetivamente disponível para ele. A via comportamental também irá ganhar repercussão na vida intrauterina, então, se espera que a mãe faça de seu comportamento uma marca que não seja traumática (WHILHEM, 2006). Assim, a influência ambiental pode iniciar-se numa etapa muito precoce, determinando se, futuramente, a pessoa, ao buscar a confirmação de que a vida vale a pena, irá à procura de experiências, ou se retrairá, fugindo do mundo(WINNICOTT, 1974).

Depois de um "primeiro despertar" na vida intrauterina, algum tempo antes do nascimento, ocorre o "grande despertar", em que o bebê está pronto para nascer (DIAS, 2003). Assim que o bebê nasce, ele depende "fundamentalmente da presença de um ambiente facilitador que forneça cuidados suficientemente bons" (DIAS, 2003, p.96). Este ambiente facilitador frente ao nascimento do bebê corresponde à condição materna de ser uma mãe suficientemente boa. Essa mãe tem como uma de suas tarefas exercer a sua função de holding para com o bebê, ou seja, dar uma sustentação adequada, que ajudará no processo de integração do lactante (CELERI, 2007). O holding diz respeito ao colo físico e psicológico, que defende o bebê de intrusões do ambiente (GRANATO, 2000). Essa modalidade de defesa protege o bebê de um retorno a um estado não integrado, do predomínio de estados de confusão psíquica, protegendo-o, portanto, de danos reais e fantasiados, e facilitando os processos de integração (GRANATO, 2000). Além disso, o holding se refere a uma "contenção física com seu substrato emocional. É o colo, o modo de envolver o bebê com cuidado, atenção e apoio afetivo" (SONAGLIO, 2009, p.120). Outra função que a mãe deverá exercer junto a seu bebê é o handling, o qual envolve "toda a sorte de cuidados físicos dispensados ao bebê, que propicia a integração psicossomática" (DIAS, 2003, p.17). Portanto, a qualidade do envolvimento materno tem importante e inegável repercussão no manejo de situações cotidianas no convívio com seu filho e na inscrição de vivências que servirão de alicerce ao psiquismo infantil.

Por fim, cabe, também à mãe a condição de apresentar os objetos à criança, ou seja, passa pelo exercício da função materna a oferta de condições para que ocorra uma "situação em que o quê, o como, o quando e o quanto a realidade externa é apresentada ao bebê são fundamentais, ocasião em que poderá utilizar seu potencial criativo, caso a mãe se ofereça como filtro que simplifica e adapta a realidade às suas necessidades e habilidades" (GRANATO, 2000, p.17).

Em casos do fenômeno de negação não psicótica da gravidez, se fazem presentes condições que podem levar à impossibilidade de recursos para que se estabeleçam o holding, o handling e apresentação de objetos adequados ao bebê. Cabe uma reflexão a respeito dos comprometimentos emocionais gerados por essas falhas no processo de integração e amadurecimento pessoal do bebê, bem como sobre as dificuldades que podem se manifestar na relação desta mãe com o seu bebê.

\section{Considerações finais}

Apesar da gravidade de efeitos decorrentes do fenômeno da negação não psicótica da gravidez, constatou-se a existência de poucos artigos científicos tanto na produção científica nacional, quanto internacional, a respeito dessa situação. Considerando ser inegável a presença desse fenô- 
meno na clínica médica e a necessidade de buscar melhor compreensão sobre a complexidade de fatores envolvidos nessa situação, o presente artigo buscou trazer à luz o tema e propor algumas reflexões sobre ele. Trata-se de uma situação não rara, na qual tanto a mãe quanto seu filho situam-se frente a riscos físicos e emocionais.

O fenômeno da negação não psicótica da gravidez interroga e solicita um olhar mais amplo, que não esteja restrito apenas a uma questão obstétrica, mas também contemple um questionamento a respeito das consequências psíquicas decorrentes de falhas na relação mãe-bebê desde a vida intrauterina. Sabe-se da relevância de que, mesmo antes do nascimento, a mulher possa ocupar-se das importantes demandas decorrentes da condição de estar gestando uma criança.

Os aportes de Winnicott a respeito dos tempos iniciais da relação mãe-bebê trazem à cena estas questões emocionais vinculadas à construção dessa relação e contribuem para uma reflexão sobre a necessidade e a urgência de buscar um estudo aprofundado sobre essa problemática. Logo, reafirma-se a relevância do Programa de Humanização no Pré-natal e Nascimento proposto pelo Ministério da Saúde. O tema da humanização requer considerar e atender demandas do período gestacional e no nascimento que assegurem melhores recursos para os dois protagonistas de uma relação singular e inaugural da condição humana.

\section{Referências}

BEYER, K.; MACK, S.M.; SHELTON, J.L. Investigative analysis of neonaticide. Criminal Justice and Behavior, v. 35, p.522-536, 2010.

BONNET, C. Adoption at birth: Prevention against abandonment or neonaticide. Child Abuse \& Neglect, v.17(4), p. 501-513, 1993.

CELERI, E.H.R.V. O livro de ouro da psicanálise. Rio de Janeiro: Ediouro; 2007.

DIAS, E.O. A Teoria do amadurecimento de D.W. Winnicott. Rio de janeiro: Imago; 2003.

DOBSON, V.; SALES, B. The science of infanticide and mental illness. Psychology, Public Policy, and Law, v.6, 1098-1112, 2000.

FERRAGU, Gorre. Le déni de grossesse : une revue de litterature. 2002. Tese (Doutorado em Psiquiatria) - Université de Rennes I : Rennes, 2002.

FRIEDMAN, S.H.; HENEGHAN, A.; ROSENTHAL, M. Characteristics of women who deny or conceal pregnancy. Psychosomatics, v.48, p. 117-122, 2007.

GRANATO, T.M.M. Encontros terapêuticos com gestantes à luz da preocupação materna primária. 2000. Tese. (Doutorado em Psicologia) - Universidade de São Paulo, São Paulo. 2000.

HALPERN R.; BARROS F.C.; VICTORIA, C.G.; TOMASI, E. Atenção pré-natal em Pelotas, Rio Grande do Sul, Brasil. Caderno de Saúde Publica v.14(3): 487-492, 1998.

MELLO, Renata Machado. A confiança na construção dos vínculos objetais: uma perspectiva psicanalítica. 2008. Dissertação. (Mestrado em Psicologia) - Universidade Federal do Rio de Janeiro, Rio de Janeiro, 2008.

MOREIRA, J.O. A ruptura do continuar a ser: o trauma do nascimento prematuro. Mental, v.5, p. 91-106, 2007. 
MINISTÉRIO DA SAÚDE. Programa de humanização no pré-natal e nascimento. Brasília: Secretaria de Políticas de Saúde (2000).

NEIFERT L.; BOURGOIS, J.A. Denial of Pregnancy a case study and literature review. Military Medicine v.165, p. 556-568, 2000.

OXFORD. Oxford advanced learner's dictionary. Oxford: Oxford University Press (2007).

PITT, S.E.; BALE, E.M. Neonaticide, infanticide, and filicide: A review of the literature. Bulletin of the American Academy of Psychiatry and the Law, v.23, 375-386, 1995.

RESNICK, P.J. Child murder by parents: a psychiatric review of filicide. American Journal of Psychiatry v.126, p. 325-334, 1969.

RESNICK, P.J. Murder of the newborn: A psychiatric review of neonaticide. American Journal of Psychiatry v.126, p.1414-1420, 1970.

SONAGLIO, H. (2009). Refletindo sobre Holding, Continência e Rêverie na entrevista de triagem: a importância desse primeiro encontro frente à dor e o desamparo. CEAPIA- Revista de Psicoterapia da Infância e da Adolescência, v.18 (18), p. 114-133, 2009.

SPIELVOGEL, A.M ; HOHENER H.C. Denial of pregnancy: a review and case reports. Birth v. 22(4), p. 220-226, 1995.

TREVISAN, M.R.; DE LORENZI, D.R.S.; ARAÚJO, M.N.; ÉSBER, K. Perfil da Assistência Prénatal entre usuárias do Sistema Único de Saúde de Caxias do Sul. RBGO v.24 (5), p. 293-299, 2002.

WESSEL, J.; ENDRIKAT, J. Clinic menstruation-like bleeding during denied pregnancy. Is there a particular hormonal cause? Gynecological Endocrinology v.21(6), p. 352-359, 2005.

WESSEL, J. Denial of pregnancy: characteristics of woman at risk. Acta Obstetricia et Gynecoligica Scandinavica v.86, p. 542-546, 2007.

WESSEL, J.; ENDRIKAT, J.; BUSCHER, U. Frequency of denial pregnancy: results and epidemiological significance of a one-year prospective study in Berlin. Acta Obstetricia et Gynecologica Scandinavica v.81, p.1021-1027, 2002.

WILHELM, J. O que é psicologia pré-natal. São Paulo: Casa do Psicólogo; 2006.

WINNICOTT,D.W. Textos selecionados: da pediatria à psicanálise (pp.49-498). São Paulo, Livraria Francisco Alves; 1950/1993.

WINNICOTT, D.W. Explorações psicanalíticas. Porto Alegre: Artes Médicas; 1974/1994.

WINNICOTT, D.W. O ambiente e os processos de maturação: estudos sobre a teoria do desenvolvimento emocional. Porto Alegre: Artes Médicas; 1979/1993 3ed.

WINNICOTT, D.W. Natureza humana. Porto Alegre: Artes Médicas; 1988/1993. 